\title{
A Digital multiple beam forming for phased array RADARs with parallel array processing
}

\author{
Mr.Nitesh Gaikwad ${ }^{(1)}$, Mr. Radha Krishna AN ${ }^{(2)}$ \\ Faculty of Electronics \& Communications Engineering. \\ Annamacharya institute of Technology \& Science,Hyderabad ${ }^{(1)}$ \\ Brilliant Group of Technical Institutions, Hyderabad ${ }^{(2)}$.
}

\begin{abstract}
Phased array radar is very important in modern radar development, and multiple digital beams forming technology is the most significant technology in phased array radar. Beam forming is a signal processing technique used in antenna arrays for directional signal transmission or reception. Digital multiple beam forming on each antenna element about large phased array radar is impossible in processor based digital processing units, because it needs simultaneous processing many A/D channels. In this project we resolve this problem by using a multi array based beam forming technique with multiplexed signal processing unit on FPGA. The conventional technique of completely duplicated hardware and also dynamic reconfiguration does not yield the real time parallel beam processing. The proposed technique employs multiplexed signal processing unit which is time shared for various beam formers. This technique provides simultaneous beams without any compromise on functionality.
\end{abstract}

\section{Introduction}

In antenna theory, a phased array is an array of antennas in which the relative phases of the respective signals feeding the antennas are varied in such a way that the effective radiation pattern of the array is reinforced in a desired direction and suppressed in undesired directions.

An antenna array is a group of multiple active antennas coupled to a common source or load to produce a directive radiation pattern. Usually, the spatial relationship of the individual antennas also contributes to the directivity of the antenna array. Use of the term "active antennas" is intended to describe elements whose energy output is modified due to the presence of a source of energy in the element (other than the mere signal energy which passes through the circuit) or an element in which the energy output from a source of energy is controlled by the signal input. One common application of this is with a standard multiband television antenna, which has multiple elements coupled together.

Digital multiple beam forming on each antenna element about large phased array radar is impossible in processor based digital processing units, because it needs simultaneous processing many A/D channels. In this project we resolve this problem by using a multi array based beam forming technique with multiplexed signal processing unit on FPGA. The conventional technique of completely duplicated hardware and also dynamic reconfiguration does not yield the real time parallel beam processing. The proposed technique employs multiplexed signal processing unit which is time shared for various beam formers. This technique provides simultaneous beams without any compromise on functionality.

\section{Phased Array Antenna}

Electronically-steered phased array radar is an array of antennas in which the relative phases of the respective signals feeding the antennas are varied in such a way that the effective radiation pattern of the array is reinforced in a desired direction and suppressed in undesired directions.

Phased array transmission was originally developed in 1905 by Nobel Laureate Karl Ferdinand Braun who demonstrated enhanced transmission of radio waves in one direction. During World War II, Nobel Laureate Luis Alvarez used phased array transmission in a rapidly-steer able radar system for "groundcontrolled approach", a system to aid in the landing of aero planes in Britain. At the same time GEMA in Germany built the PESA Mammut. The design is also used in radar, and is generalized in inter-ferometric radio antennas. In 2007 DARPA researchers announced a 16 element phased array integrated with all necessary circuits to send at $30-50 \mathrm{GHz}$ on a single silicon chip for military purposes.

These antenna arrays completely consist of singles radiating elements and each of it gets an own phase shifter. The elements are ordered in a matrix array. The planer arrangement of all elements forms the complete phased array antenna. One of the advantages is the Beam forming in two planes are digital Beam forming is possible. 


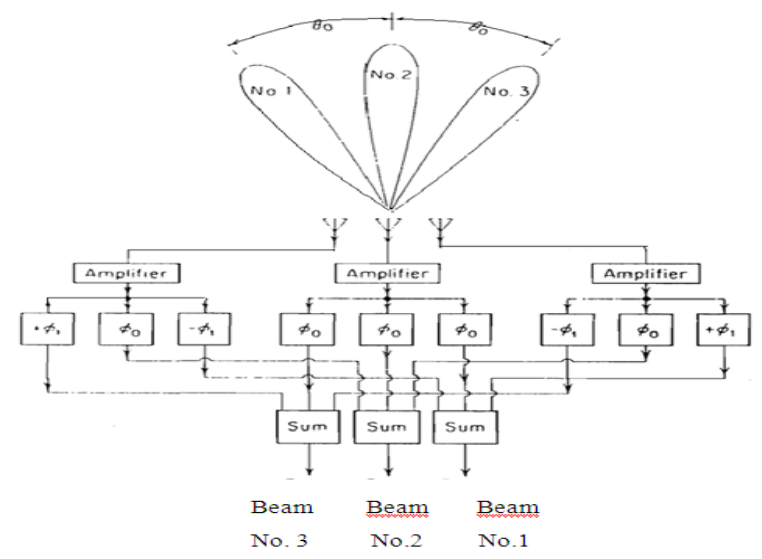

Fig 2.1: Electronic phased array beam formation

\section{RF Translator}

\section{Hardware Specifications}

Radio frequency (RF) is a rate of oscillation in the range of about $3 \mathrm{kHz}$ to $300 \mathrm{GHz}$, which corresponds to the frequency of radio waves, and the alternating currents which carry radio signals. RF usually refers to electrical rather than mechanical oscillations, although mechanical RF systems do exist.

In order to receive radio signals an antenna must be used. However, since the antenna will pick up thousands of radio signals at a time, a radio tuner is necessary to tune in to a particular frequency (or frequency range).This is typically done via a resonator - in its simplest form, a circuit with a capacitor and an inductor forming a tuned circuit.

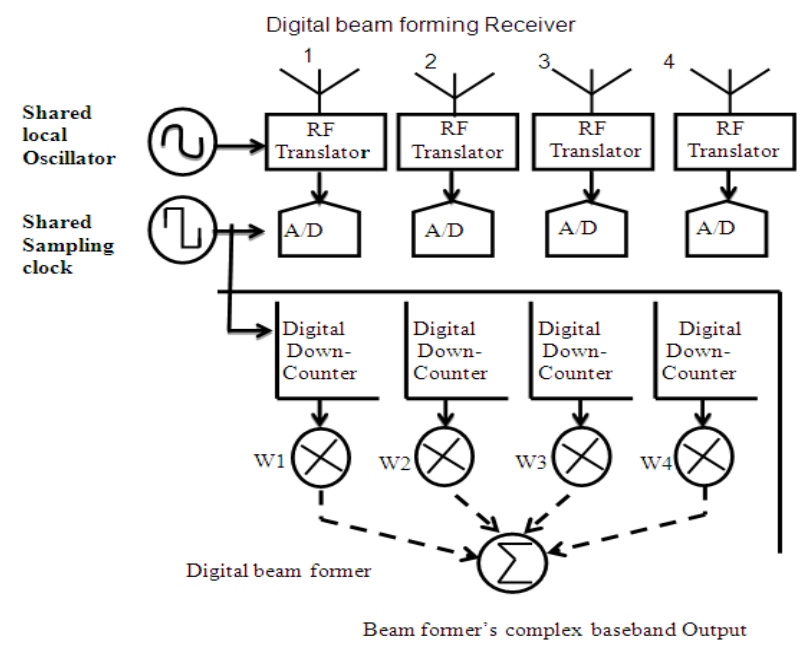

Fig 3.1 Digital Beam Forming

The circuit amplifies oscillations within a particular frequency band, while reducing oscillations at other frequencies outside the band.

\section{Digital Down-Counter}

The principle of Digital Down-Counter which is taking the input from RF Translator that is given to the input of Fast ADC . The output of Fast ADC block which is given to the DDC block. The DDC (Digital DownCounter) consists of NCO (Numerically Controlled Oscillator), Multiplier, low pass filter and Decimator. 

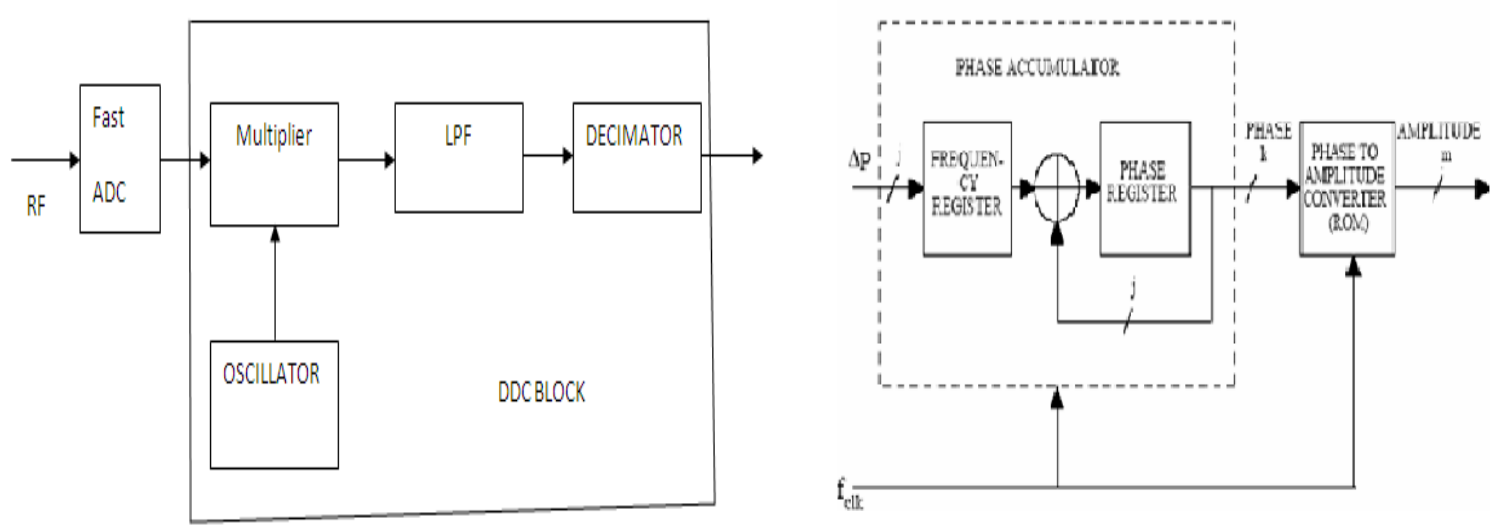

Fig.2.2 Digital dorn Counter

Fig.3.3. NCO (Oscillator)

\section{Oscillator (NCO)}

The NCO main purpose is to generation the carrier signals (cosine). The main advantage is ROM based techniques will be used for area optimization.

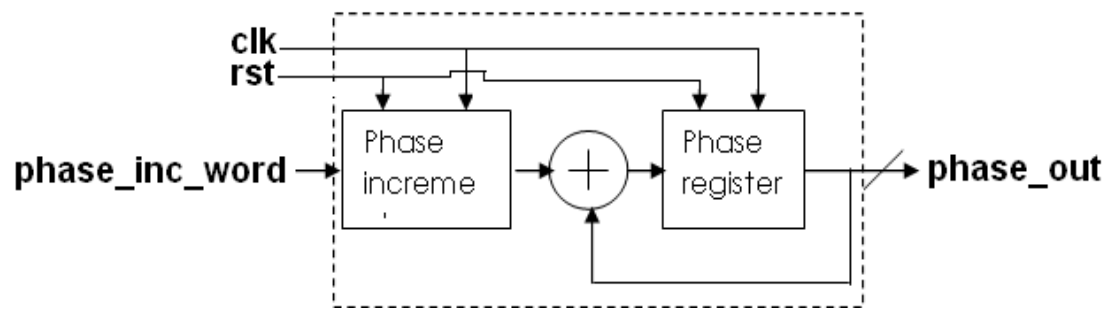

Fig.3.4 phase accumulator

The output of phase accumulator when the phase increment value is 0000001000000000 . It can be observed that the resulting phase value after each clock pulse is four added to the previous phase value. In the following figure initial phase is 0 and further with clock pulses resulting in $4,8,12$, and 16 ...The output of phase accumulator is added with multiplier output. That output is given to NCO block.

All the blocks are connected with common clock and reset signals. The delta phase value decides the phase increment for each clock pulse. Hence decides the resulting signal frequency. The Frequency modulating instantaneous value is added to the delta phase value which causes instantaneous change in frequency. Due to the digital nature of the modulator only at each clock tick the modulating signal value shall affect the resulting frequency. If the modulating signal is analog then an Analog Digital converter must be used to digitize the modulating signal which can be used in NCO.

\section{Low Pass Filter}

A low-pass filter is an electronic filter that passes low-frequency signals but attenuates (reduces the amplitude of) signals with frequencies higher than the cutoff frequency. The actual amount of attenuation for each frequency varies from filter to filter. It is sometimes called a high-cut filter, or treble cut filter when used in audio applications. A low-pass filter is the opposite of a high-pass filter. A band-pass filter is a combination of a low-pass and a high-pass. 


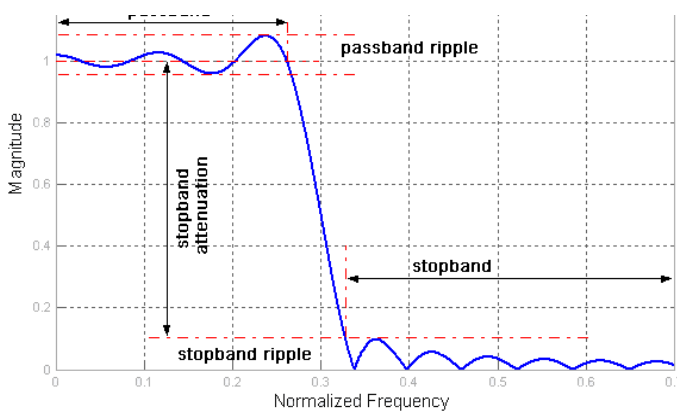

Fig 3.5: Low Pass Filter

\section{Decimator (Filtering \& Decimation)}

There are two main classes of DDC - wideband and narrowband, differentiated by their decimation ratios. As a rough guide, if the decimation ratio is less than 32 consider the DDC wideband; if 32 or more, the DDC is narrowband.The filtering we will perform is different for narrowband or wideband, so is tackled separately. However, the decimators can be treated identically for wideband or narrowband systems.

\section{Designing the Filters}

There can be several tools by which the filter coefficients can be finalized for implementing the required FIR filters. In this project the MATLAB's filter design analysis tool (FDA) will be used for designing.

An FIR filter is one whose impulse response is of finite duration. In this filter, the current output $\left(\mathrm{y}_{\mathrm{n}}\right)$ is calculated solely from the current and previous input values $\left(x_{n}, x_{n-1}, x_{n-2} \ldots\right)$. This type of filter is also said to be non-recursive.

The difference equation, which defines how the input signal is related to the output signal

$$
y(n)=b_{0} x(n)+b_{1} x(n-1)+\cdots+b_{P} x(n-P)
$$

Where $\mathrm{P}$ is the filter order, $\mathrm{x}(\mathrm{n})$ is the input signal, $\mathrm{y}(\mathrm{n})$ is the output signal and $\mathrm{b}_{\mathrm{i}}$ are the filter coefficients. The previous equation can also be expressed as

$$
y(n)=\sum_{i=0}^{P} b_{i} x(n-i)
$$

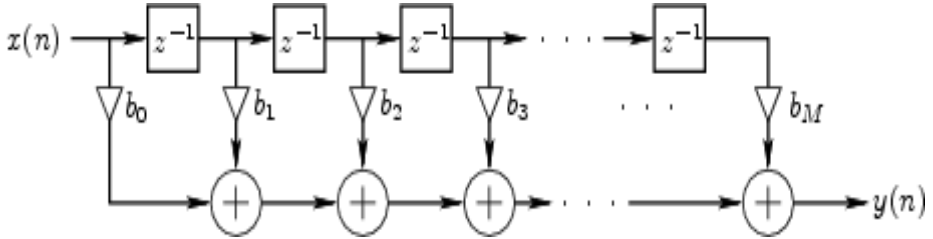

Fig 3.6 Finite Impulse Response Digital Filters

The transfer function allows us to judge whether or not a system is BIBO stable. To be specific the BIBO stability criterion requires all poles of the transfer function to have an absolute value smaller than one. In other words all poles must be located within a unit circle in the $z$-plane.

\section{Multiplier \& adder}

Here, The output generated by DDC which is multiplied with the generated weights. This output is given to next module as adder which will add all the 16 signals to generate a beam.

VIII. Specifications

Table 1 Beam forming specifications

\begin{tabular}{|c|c|c|}
\hline Parameter & Value & Comments \\
\hline Input signal type & Band pass signal & Coming from typical superhytrodyne receiver \\
\hline Input signal frequency range & $1.5-3.5 \mathrm{MHz}$ & $2 \mathrm{MHz} \mathrm{BW}$ and $2.5 \mathrm{Mhz}$ IF frequency value \\
\hline Sampling rate & $10 \mathrm{MHz}$ & \\
\hline DDC filter size & 16 taps & DDC output sampling rate $2.5 \mathrm{Msps}$ \\
\hline DDC decimation & 4 & As fo=fs/4 \\
\hline NCO values: COS & $10-10$ & As fo=fs/4 \\
\hline NCO value : - SIN & $0-101$ & Linear array as shown in below figure. \\
\hline Number of array elements & 16 & d as shown in below figure \\
\hline Element spacing & 3 meters &
\end{tabular}


Table 2 True Angle values of beam

\begin{tabular}{|l|l|}
\hline DDS Value & Angle \\
\hline 8 & 11.25 \\
\hline 20 & 28.125 \\
\hline 80 & 112.5 \\
\hline 128 & 180 \\
\hline
\end{tabular}

\section{Spatran 6e}

The Spartan®-6 families provides leading system integration capabilities with the lowest total cost for high-volume applications. The thirteen-member family delivers expanded densities ranging from 3,840 to 147,443 logic cells, with half the power consumption of previous Spartan families, and faster, more comprehensive connectivity. Built on a mature $45 \mathrm{~nm}$ low-power copper process technology that delivers the optimal balance of cost, power, and performance, the Spartan-6 family offers a new, more efficient, dualregister 6-input lookup table (LUT) logic and a rich selection of built-in system-level blocks. These include $18 \mathrm{~Kb}(2 \times 9 \mathrm{~Kb})$ block RAMs, second generation DSP48A1 slices, SDRAM memory controllers, enhanced mixed-mode clock management blocks, SelectIO ${ }^{\mathrm{TM}}$ technology, power- optimized high-speed serial transceiver blocks, PCI Express ${ }^{\circledR}$ compatible Endpoint blocks, advanced system-level power management modes, auto-detect configuration options, and enhanced IP security with AES and Device DNA protection. These features provide a low- cost programmable alternative to custom ASIC products with unprecedented ease of use. Spartan-6 FPGAs offer the best solution for high-volume logic designs, consumer-oriented DSP designs, and cost-sensitive embedded applications. Spartan-6 FPGAs are the programmable silicon foundation for Targeted Design Platforms that deliver integrated software and hardware components that enable designers to focus on innovation as soon as their development cycle begins.

The design is functionally verified by simulating the code in ModelSim from Mentor Graphics. The FPGA synthesis is done using Xilinx ISE tool. The synthesis results of ISE are analyzed for timing and area. The hardware output i.e. SPARTAN 6E FPGA output shows on Chip scope pro analyzer.

\section{Results}

The simulation and chip scope waveforms of beam forming which will show the maximum gain in desired direction and minimum gain in the unwanted direction.

1. Device utilization summary

Selected Device

Slice Logic Utilization

Number of Slice Registers

Number of Slice LUTs

Number used as Logic

Number used as Memory

Number used as RAM

Number used as SRL

Number of unique control sets

IO Utilization

Number of IOs

Number of bonded IOBs

IOB Flip Flops/Latches

Specific Feature Utilization:

Number of Block RAM/FIFO

Number using Block RAM only

Number of BUFG/BUFGCTRLs

Number of DSP48A1s

Total REAL time to execution completion

Total CPU time to execution completion

Total memory usage is 191484 kilobytes

Number of errors

Number of warnings

Number of infos 6slx $16 \operatorname{csg} 324-3$

2840 out of $18224 \quad 15 \%$

8139 out of $911289 \%$

7450 out of $9112 \quad 81 \%$

689 out of $2176 \quad 31 \%$

180

509

183

58

58 out of $232 \quad 25 \%$

28

28 out of $\quad 32 \quad 87 \%$

28

8 out of $\quad 16 \quad 50 \%$

12 out of $\quad 32 \quad 37 \%$

45.00 secs

44.47 secs

0 ( 0 filtered)

526 ( 0 filtered)

37 ( 0 filtered) 
2. Simulation results of antenna array module

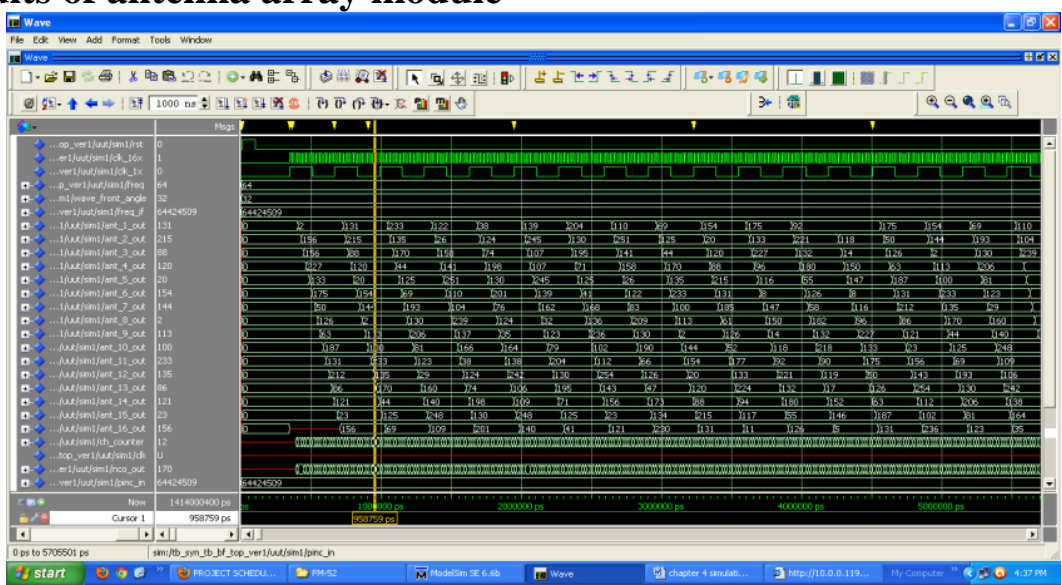

Fig 5.1 Antenna Array Digital outputs

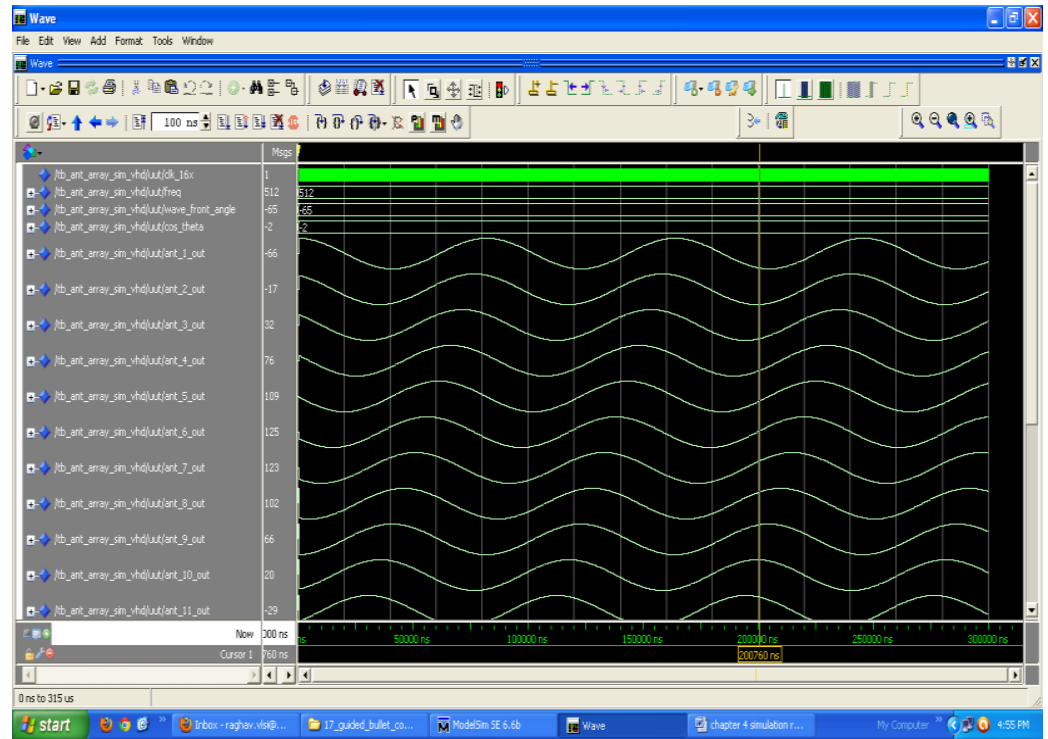

Fig 5.2 antenna array outputs with phase shifts

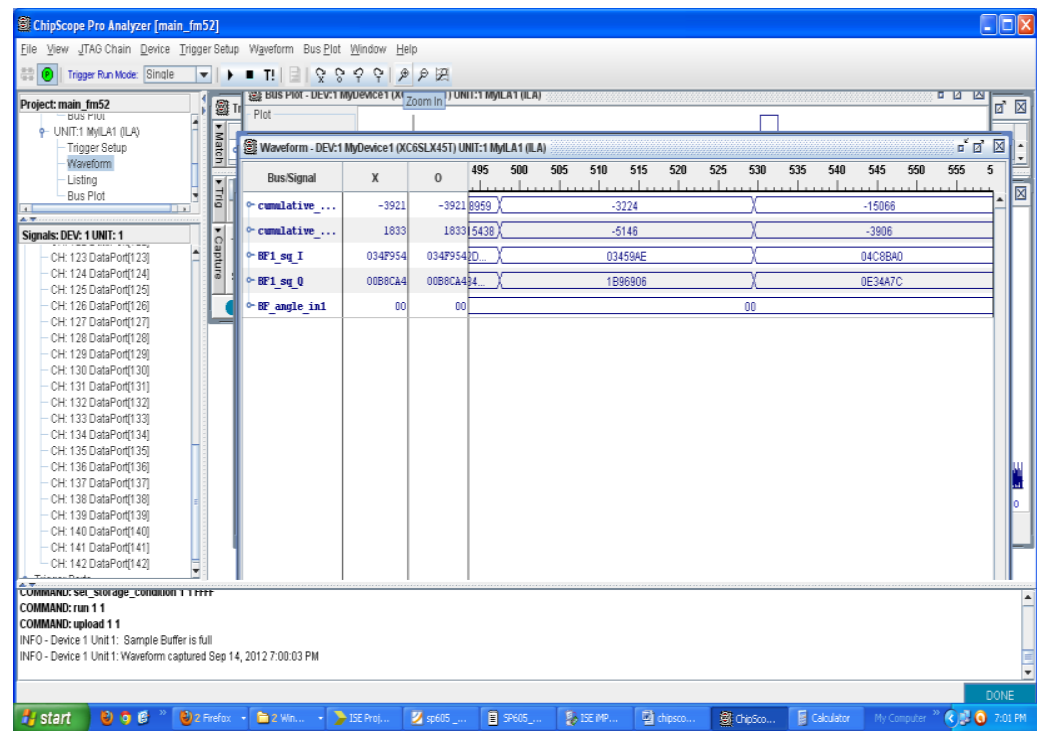

Fig 5.3 Beam forming digital I \& q square signals 


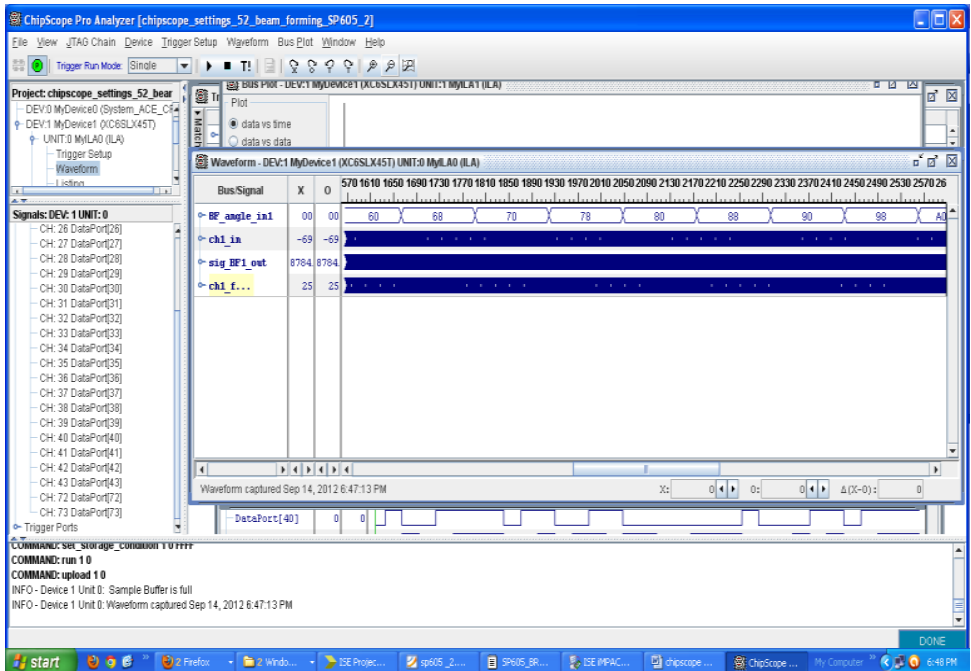

Fig 5.4 Beam forming digital output

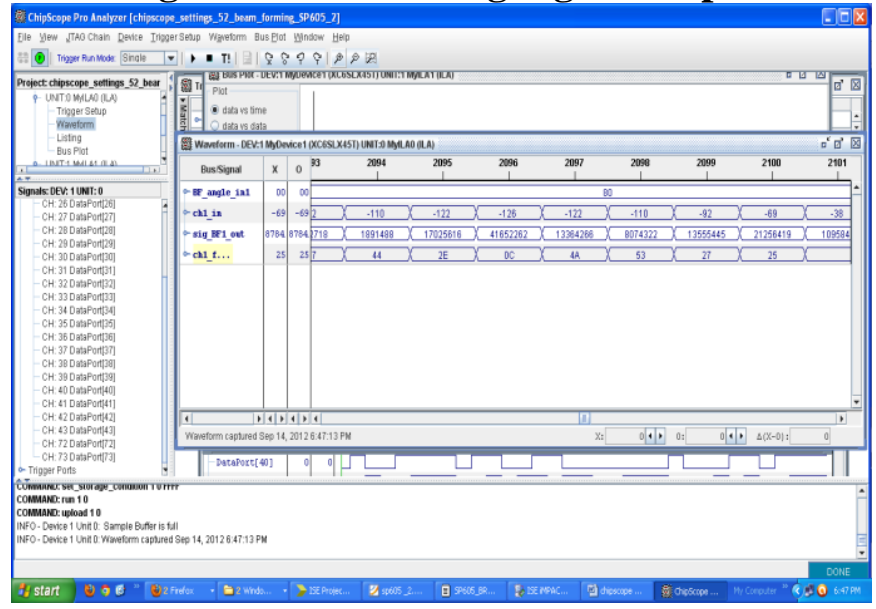

Fig 5.5 Chip scope digital output

\section{Conclusion}

In this project has explored the design and implementation of a low-cost digital beam forming platform. The proposed technique employs multiplexed signal processing unit which is time shared for various beam formers. This technique provides simultaneous beams without any compromise on functionality. The low cost of the system facilitates its easy integration into phased array radar systems. The process of beam forming implies weighting these digital signals. In this project, by using 16 antenna input signals we generated digital multiple beams with parallel array processing and we can implement more than 16 antenna input signals. Here we designed the digital beam forming by using the sampling frequency and the digital baseband signals then represent the amplitudes and phases of signal received at each element of the array. The process of beam forming implies weighting these digital signals.

\section{References}

[1] A dynamically Reconfigurable Phased Array Radar Processing System,Emmanuel Seguin, Russell Tessier, EricKnapp, and Robert W.Jackson

G. W. Stimson: "Introduction to Airborne Radar, 2nd Ed.," SciTech Publishing, 1998

[3] P. Lacombe, J.-P. Harding, J.-C. Marchais, E. Normant: "Air and Spaceborne Radar Systems: An Introduction," IEE, 2001

[4] M. I. Skolnik: "Introduction to Radar Systems, 3rd Ed.," McGraw-Hill, 2005

[5] R. J. Mailloux: "Phased Array Antenna Handbook," Artech House, 2005

[6] E. Brookner: "Practical Phased Array Antenna Systems," Artech House, 1991

[7] R. C. Hansen: "Phased Array Antennas," John Wiley \& Sons, 1998

[8] A. Ludloff: "Praxiswissen Radar und Radarsignalverarbeitung, 2. Auflage," Viewegs Fachbücher der Technik, 1998. 